\title{
QPSK Demodulator based on a New Half Mode Substrate Integrated Waveguide Six-port Junction
}

\author{
S. Lakhdhar, F. Harabi and A. Gharsallah \\ Research in High Frequency Electronic Circuits and Systems, Faculty of Mathematical, Physical and \\ Natural Sciences of Tunis, El Manar University, 2092,Tunisia saoussenlakhdhar@gmail.com, \\ ferid.harabi@gmail.com, ali.gharsallah@gmail.com
}

\begin{abstract}
Objectives: This study presents a novel architecture of a six-port demodulator operating at $2.45 \mathrm{GHz}$. The new structure of the six-port junction was based on a branch-line coupler and made up of a Wilkinson power divider and three $90^{\circ}$-hybrid coupler.

Methods: The six-port design was accomplished using HMSIW technology. Simulation was carried out using ADS software of Agilent technologies and HFSS software. Then, an RF-DC conversion circuit was optimized and designed. Our choice fell on a Schottky diode-based power detector and a resistive adaptation to its input. Subsequently, to validate this system we have assembled these two circuits using a co-simulation Harmonic Balance-momentum. Then the six-port configuration was connected to power detectors to show its performance as a demodulator circuit. Finally, the use of an appropriate algorithm on MATLAB allowed us to extract the amplitudes of the four output signals as a function of the phase difference between the two input signals.

Findings: The obtained results showed that the six-port junction has good performances in terms of phase difference, return loss, reflection and isolation. We conclude that the six-port junction, designed as a phase discriminator, has the best qualities for demodulating an RF signal.

Application: For each output voltage we have a phase shift for which the corresponding power is canceled so the power detector allows, by measuring the output powers and by detecting the one that is minimal compared to the others, to differentiate the four states of modulation which highlights the good behavior of our system.
\end{abstract}

Keywords: Six-port junction, HMSIW, Power detector, Linearization, QPSK demodulation.

\section{Introduction}

Taking advantages of developments in the fields of integrated circuits and microwaves, the six-port technique

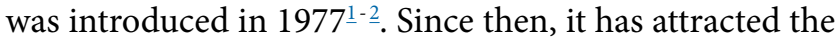
interest of academic and scientific research and has been the subject of numerous studies. Throughout its development, the six-port technology has invested several application areas such as radar systems, RF signal arrival direction determination systems and homodyne demodulators. In the field of telecommunications, the six-port technique has been successfully implemented in wireless reception systems. New six-port direct-conversion architecture has replaced conventional heterodyne and homodyne architectures by minimizing the complexity, cost, and consequently power consumption of such a system. Its potential is particularly considered in its ability to perform the direct demodulation of QPSK modulated signals. The idea of using six-port as a measurement technique was considered as a solution to be able to measure high 
frequency voltage and current. The proposed method consists in measuring the waves transmitted and reflected at each port, the dispersion matrix then contains this information.

Six-port reflectometry is one of the first applications developed in this field. The six-port reflectometer is a microwave measurement device that can be used to determine the reflection coefficient of a device or alternatively to find the ratio in modulus and phase between two different signals ${ }^{-\underline{4}}$. It is a passive and linear circuit consisting of two input ports one is connected to an RF source and the other one is connected to the device to be characterized, and four output ports connected to four power detectors. Because the structure of an SPR is much simpler than an automatic network analyzer, it is a good candidate for a monolithic realization. In addition to its important role in the fields of metrology, the six-port technique has also been used successfully in radar systems to determine the speed and direction of targets. There are different radar systems, such as pulse radars, FM-CW radars and duplex radars ${ }^{5-6}$. In order to obtain the speed and the direction of the target, it makes it possible to measure the shifts of the Doppler frequency which is the frequency difference between the waves emitted by radar and the waves received after reflection on the targets. The introduction of the six-port technique in the field of tele communications begin with the work. He proposed à new concept of direct conversion digital receiver based on a six-port network.

In 1995, he developed a study in which he compared the performance of a SPR and a classical IQ demodulator ${ }^{7}$. Tatu realized a six-port receiver with MHMIC technology operating in the frequency band $23-31 \mathrm{GHz}^{\underline{8}-9}$. Then several researches were made allowing a wide use of this architecture in the wireless communications market $\underline{10}-\underline{14}$. In this study, we present a new structure of a six-port junction and a power detector device to show the comportment of the system as a QPSK demodulator.

The rest of this paper is organized as follow: section 2 details the theory of the six-port demodulator. Section 3 shows the new architecture of the HMSIW junction. Section 4 presents the power detector circuit and section 5 shows simulation results due to the assemblage of the two devices.

\section{Analyses of the QPSK demodulation based on a six-port junction}

\subsection{Six-port junction theory}

The conventional six-port circuit is a passive component consisting of couplers and transmission lines. It can be considered as a black box with two inputs and four outputs. One of the two inputs is used for the signal to be characterized and the other for the reference signal. These two signals will be summed according to the architecture of the junction giving four signals to the outputs that serve to define the RF signal. The six-port junction to be studied consists of three $90^{\circ}$ hybrid couplers and a Wilkinson power divider. This structure is specifically designed for the demodulation of a QPSK modulated RF signal. Figure 1 illustrates the organization of a modified six-port junction. We can show that all ports 1 to 4 combine the input signals differently. Then knowing the reference signal from the oscillator LO, we can characterize the phase and the amplitude of the RF signal. From this circuit we can deduce the expressions of the four reflected waves, b1, b2, b3 and b4, as a function of the two incident wave's a5 and a6. As shown in Figure 2 the reflected wave at each output port of the six-port junction can be written as:

$$
\begin{aligned}
& b_{1}=-j \frac{a_{5}}{2}+j \frac{a_{6}}{2} \\
& b_{2}=\frac{a_{5}}{2}+j \frac{a_{6}}{2} \\
& b_{3}=\frac{a_{5}}{2}+\frac{a_{6}}{2} \\
& b_{4}=-j \frac{a_{5}}{2}-\frac{a_{6}}{2}
\end{aligned}
$$

After it has been amplified, the RF signal will be injected at one of the two inputs of a QPSK demodulator composed of a six-port junction, four RF diodes, and an I / Q decoder as shown in Figure 1. 


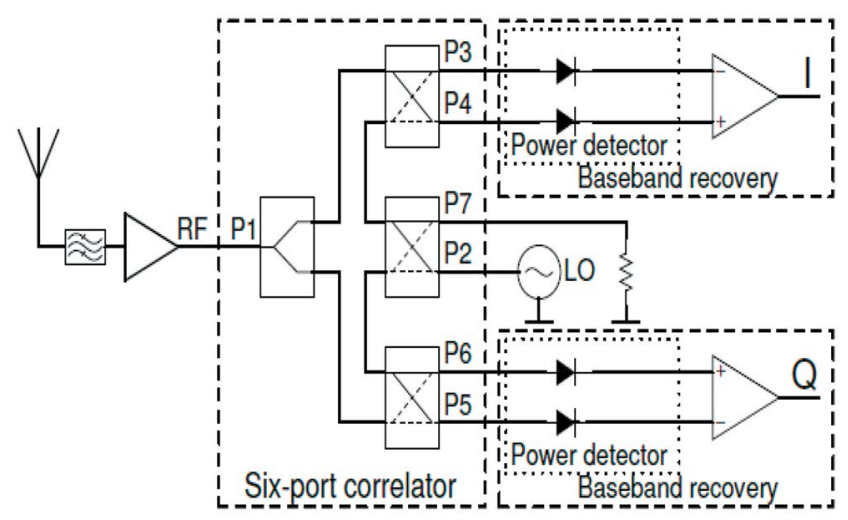

Figure 1. Six-port demodulator

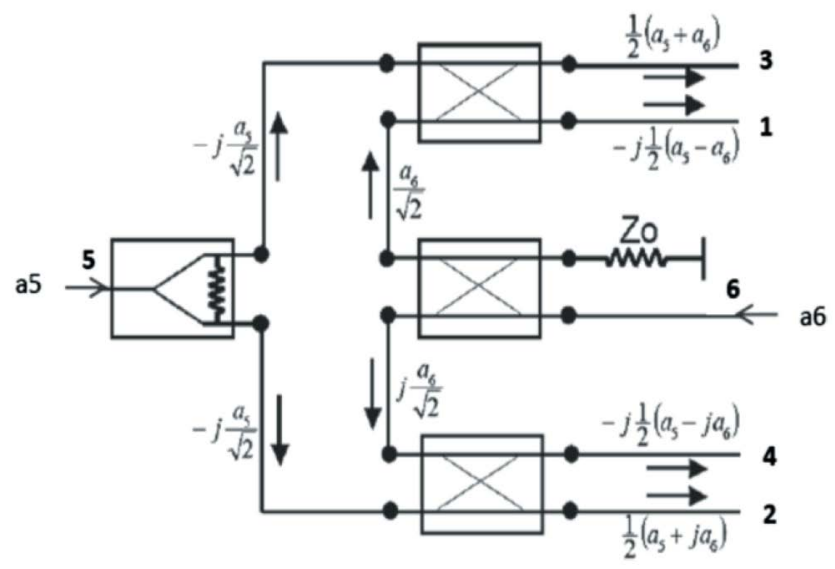

Figure 2. Six-port junction

\subsection{Six-port demodulator architecture}

If a power detector is connected to each output port of the junction, the resulting DC voltage can be expressed as:

$$
\begin{gathered}
V_{i}=k\left|b_{i}\right|^{2} \\
V_{1}=k \frac{|a|^{2}}{4}|1-\exp (j \Delta \theta)|^{2}=k|a|^{2}\left|\sin \left(\frac{\Delta \theta}{2}\right)\right|^{2} \\
V_{2}=k \frac{|a|^{2}}{4}\left|1-\exp \left(j \Delta \theta-\frac{\pi}{2}\right)\right|^{2}=k|a|^{2}\left|\sin \left(\frac{\Delta \theta-\frac{\pi}{2}}{2}\right)\right|^{2} \\
V_{3}=k \frac{|a|^{2}}{4}|1-\exp (j \Delta \theta-\pi)|^{2}=k|a|^{2}\left|\sin \left(\frac{\Delta \theta-\pi}{2}\right)\right|^{2}
\end{gathered}
$$

$$
V_{4}=k \frac{|a|^{2}}{4}\left|1-\exp \left(j \Delta \theta+\frac{\pi}{2}\right)\right|^{2}=k|a|^{2}\left|\sin \left(\frac{\Delta \theta+\frac{\pi}{2}}{2}\right)\right|^{2}
$$

Where, $\mathrm{K}$ is a constant which depends on the power detector characteristics and the gain of the baseband circuit. $\Delta \theta$ represents the phase difference between the two input waves.

From these equations we can notice that the measured power at each output port is periodic of $2 \pi$ and the four minimum values of the voltages are separated by multiples of $\frac{\pi}{4}$.

\section{HMSIW Six-Port junction}

\subsection{Design}

The six-port junction is composed of three branch line couplers and a Wilkinson power divider based on HMSIW technology. The two circuits are connected by $50 \Omega$ lines. These lines must be optimized in such a way as to have very precise lengths making it possible to obtain the results closest to the theoretical values. In fact, poor optimization of these lengths damages the operation of the entire junction. The layout of the optimized six-port junction is presented in Figure 3. We considered that ports 5 and 6 are the input ports and that ports 1 to 4 are the output ports.

\subsection{Simulation results}

Figure 4 shows the return loss and the isolation parameters at the input ports. We notice that these two ports are well matched. The value of the return loss is better than $10 \mathrm{~dB}$ in the frequency range from $2.2 \mathrm{GHz}$ to $2.6 \mathrm{GHz}$. Also we find that we have a good isolation between these two ports having a value less than $-20 \mathrm{~dB}$ in the total frequency band from $1.8 \mathrm{GHz}$ to $3.2 \mathrm{GHz}$.

Figure 5 illustrates the magnitudes of the transmission parameters $S$ between the two input ports and the four output ports. We find that these parameters are almost identical having a value around $-8 \mathrm{~dB}$ in the frequency band from $2.2 \mathrm{GHz}$ to $2.4 \mathrm{GHz}$. So we can conclude that the sharing of input signal to the output ports is perfectly realized. From Figure 6 which presents the phases of the transmission parameters between the two inputs and 


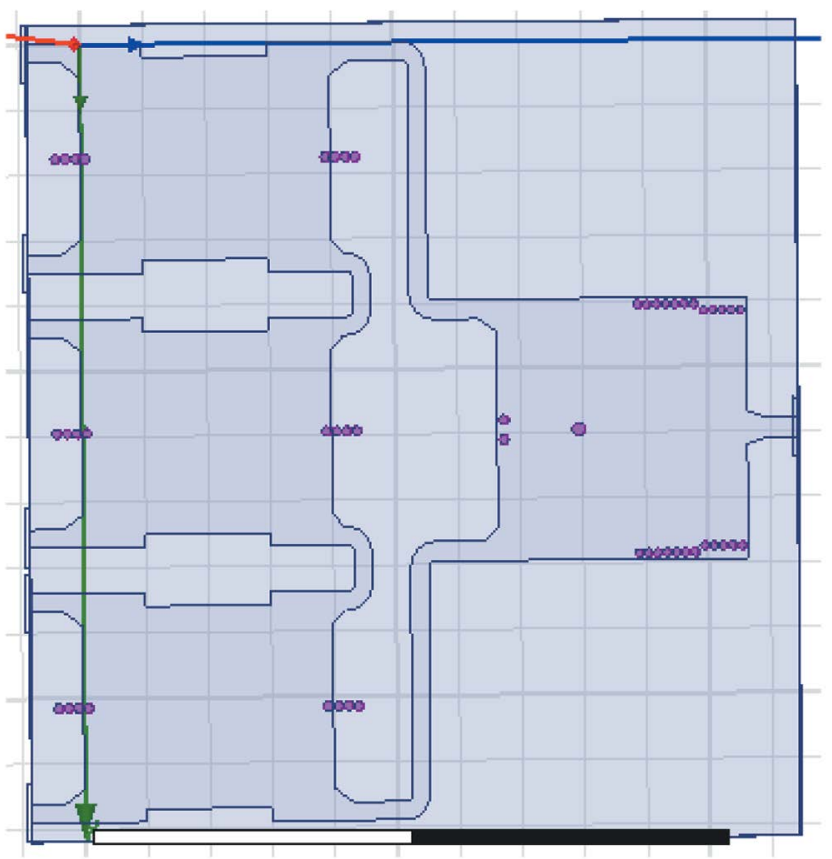

Figure 3. Layout of the Six-port junction

the four outputs, we can deduce that at the frequency $2.45 \mathrm{GHz}$ the phase differences between these ports are acceptable.

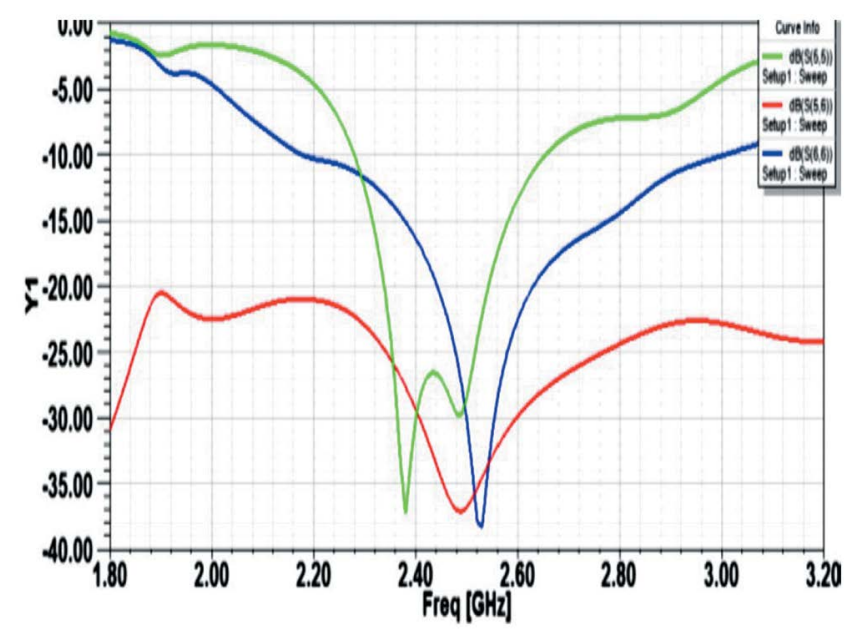

Figure 4. Reflection coefficients and isolation

\section{Power detector circuit}

\subsection{Design}

The four output signals of the six-port junction will be detected by the four power detectors which allow to reject the high frequency components and to recover
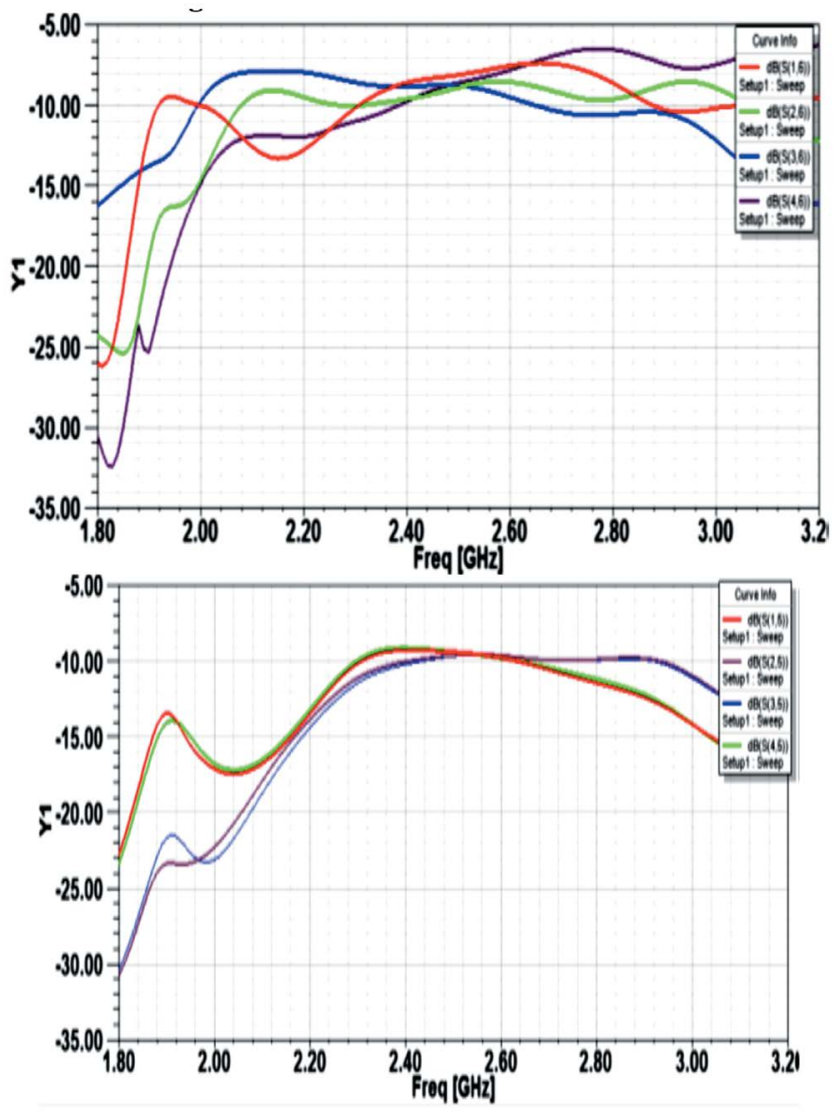

Figure 5. Reflection coefficients and isolation between the two input ports

the low frequency components. In the case of RF energy recuperation, it is important to design a high sensitivity RF-DC conversion circuit in order to have a usable amount of DC power; the sensitivity of the detector is directly related to the sensitivity of the used diodes

As shown in Figure 7, the circuit is composed of:

1. A connection capacity $C$ which permits to block any DC component generated by the diode.

2. A resistor used to adapt the detector to its input. In fact, the adaptation can be reactive using an LC or resistive circuit using a resistor 50 . The disadvantage of the resistive adaptation is the decrease of the sensitivity because of the dissipation of power in the $50 \Omega$ resistor, to overcome this problem and compensate for loss of sensitivity, very high impedance instrumentation amplifiers must be connected to the output of each detector.

3. A low-pass filter that is used to reject the HF components of the detected signal

4. Schottky diode 

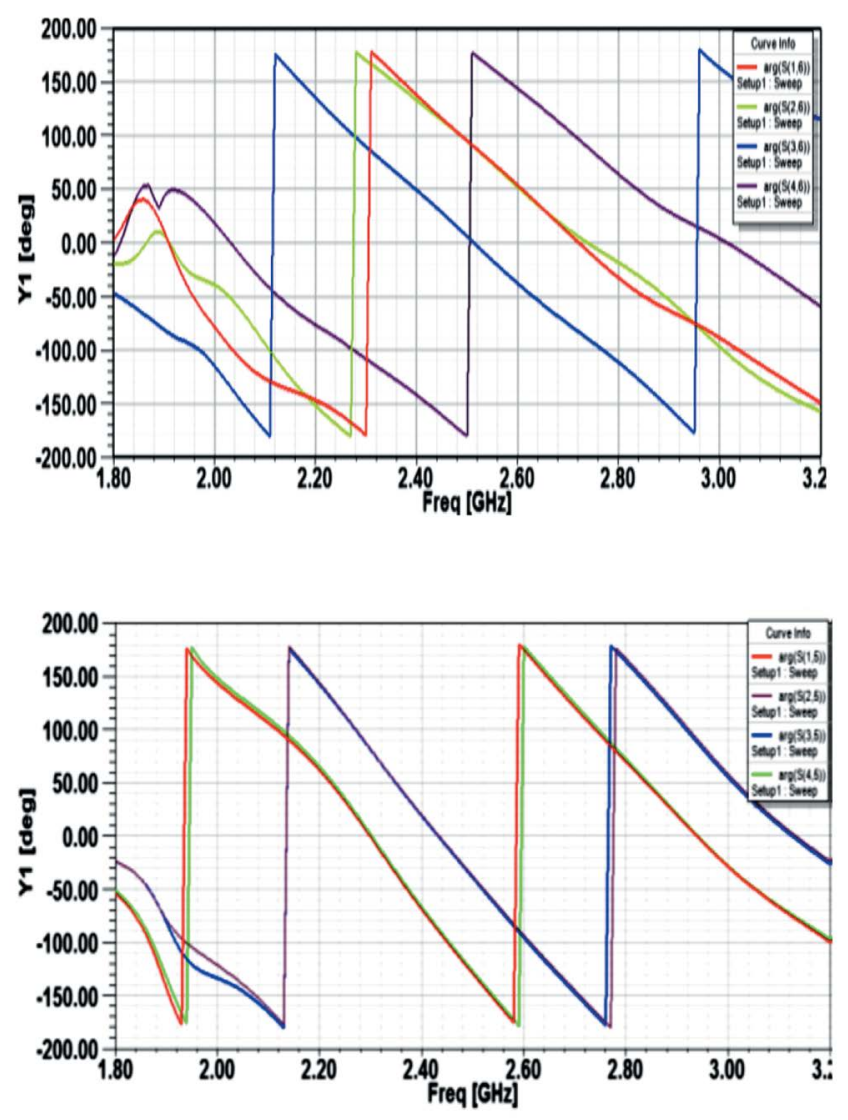

Figure 6. Phase difference between the RF input port and the four output ports

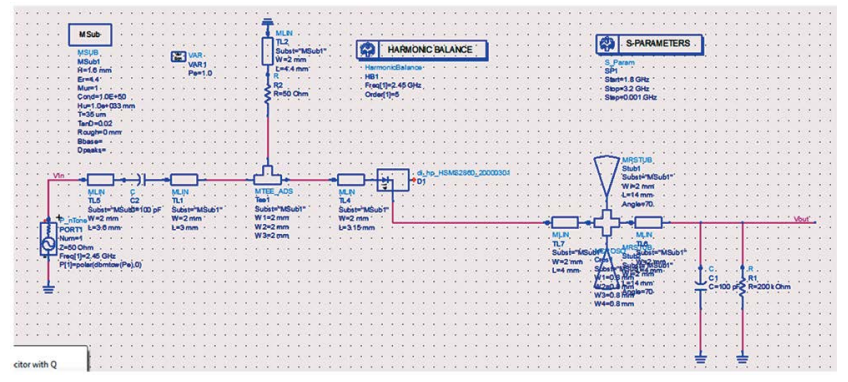

Figure 7. The power detector circuit

To improve the filtering effect of the RF signal, a butterfly stub can be placed at the output of the detector. The stub is an open circuit which eliminates residual signals at $2.45 \mathrm{GHz}$. It also makes to reduce the short-circuit on the micro-strip line using the open circuits placed at its ends.

\subsection{Power detector linearization}

We find that there are 2 modes of operation:- Pe $<-10$ $\mathrm{dBm}$ : In this case a quadratic detection is performed by the diode andthe power of the input RF signalCan be measured - Pe > $-10 \mathrm{dBm}$ : In this case the approximations made previously are no longer valid, the diode operates in switching and the device performs a conventional envelope detection. The output voltage is therefore proportional to the amplitude of the input RF signal. Therefore, we must perform a correction of the output voltage to increase the power measurement dynamics. As shown in Figure 8 access 1 of the six-port junction is connected to a RF generator which delivers a $2.45 \mathrm{GHz}$ frequency, port 2 of the six-port junction is connected to a $50 \mathrm{ohm}$ load. We simulated the system using the ADS platform and by varying the input power $\mathrm{Pe}$ of the generator from -30 $\mathrm{dBm}$ to $5 \mathrm{dBm}$ with a step of incrimination of $1 \mathrm{dBm}$. The four voltages acquired at the outputs of the detectors are processed by a MATLAB script which determines the 4 correction models.

Figure 9 shows the result of the model corresponding

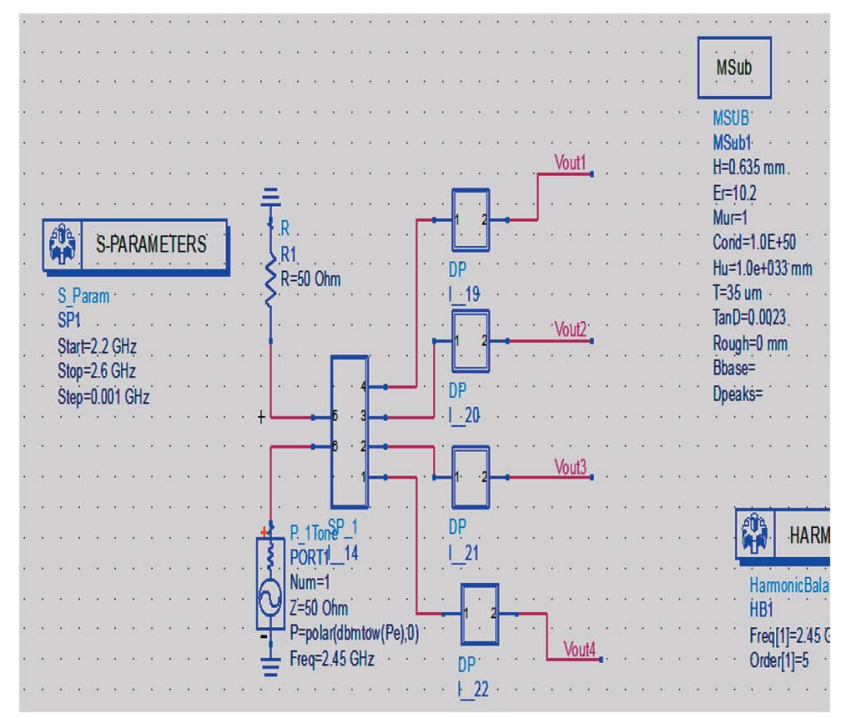

Figure 8. Linearization circuit

to the four detectors, for a polynomial of order $M=4$. It is seen that after the correction, the input power P1 and the output voltages are proportional. Also, the slope of this voltage is constant. We can clearly see that the power detector has been well linearized. The results obtained are satisfactory and show us the validity of the method

The choice of a power dynamics ranging from -30 to $5 \mathrm{dBm}$ is explained by the fact that the diodes saturate above $5 \mathrm{dBm}$ and that below $-30 \mathrm{dBm}$, the noise of the system becomes preponderant and the measurement will be imprecise. We also see that for powers between -30 
and $-15 \mathrm{dBm}$, detectors perform a quadratic detection without correction.

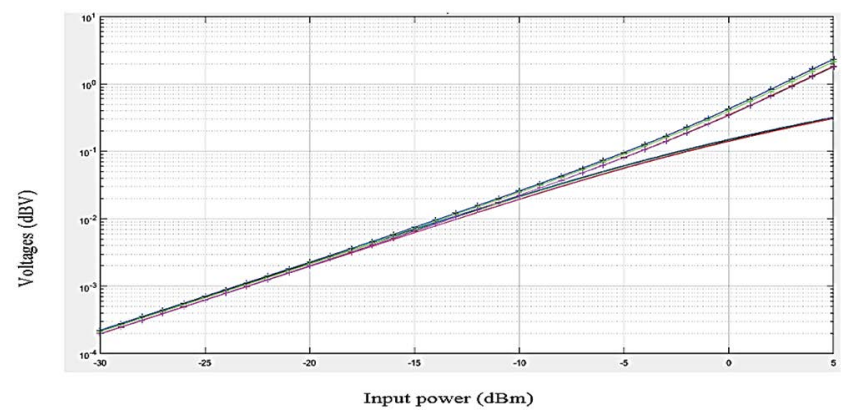

Figure 9. The output voltages before and after linearization

\section{Results and discussion}

In order to ensure the possible coupling between the various designed components and to validate the theoretical developed studies, Harmonic Balance (HB) simulations were performed. This frequency analysis method HB allows us to obtain the variations of DC voltages at the outputs of the power detectors as a function of the phase difference between the two input RF signals. Figure 10 shows the distribution of the harmonics of the output signal after detection. These harmonics are spread over a frequency band ranging from 0 to $5 \mathrm{GHz}$. We note that the maximum component of these harmonics is located at the zero frequency whereas at the frequency $2.45 \mathrm{GHz}$ the magnitude of the signal is almost equal to zero which shows a good rejection of the HF signals. In Figure 11 we have presented the measured output states by varying the phase shift. In order to take the variations of the amplitudes of the four output signals as a function of phase difference between the two input signals, we used a suitable algorithm written under. The simulation result presented in Figure 11 shows four periodic sinusoidal signals of $2 \pi$ with magnitudes of a few milli volts. We find that the four minimum voltage values are separated by multiples of $\frac{\pi}{4}$. Also for each output, there is a phase shift between the two input signals, for which the corresponding power is canceled.

\section{Conclusion}

In this study a new design of a six port junction based on HMSIW technology and a RF-DC conversion circuit
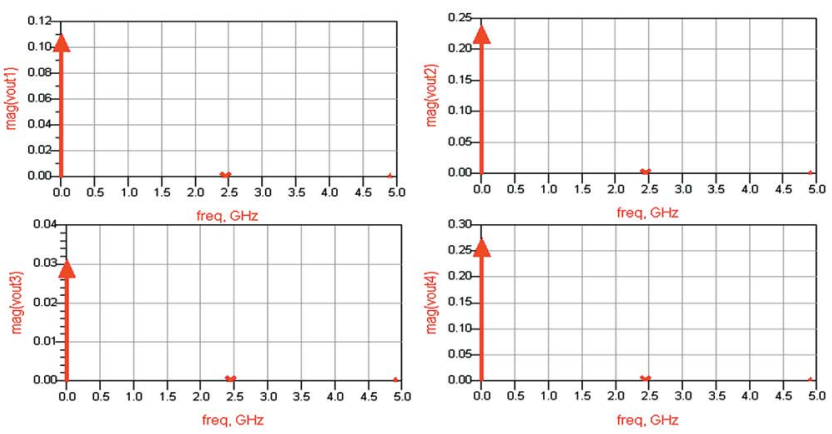

Figure 10. The output voltages for $\Delta \theta=\frac{\pi}{4}$

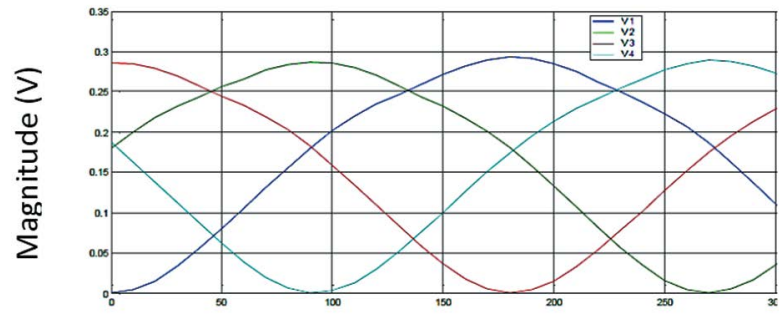

Phase(deg)

Figure 11. variations of the magnitudes of the four output signal

based on microstrip technology was presented. Then both circuits were assembled using ADS-CAO platform. Finally, the use of an appropriate algorithm on MATLAB allowed us to take the amplitudes of the four output signals as a function of the phase difference between the two input signals. For each output voltage we have a phase shift for which the corresponding power is equal to zero. So the power detector allows, by measuring the output powers and by detecting the one that is minimal compared to the others, to differentiate the four states of modulation which exhibit the good behavior of our system. We conclude, too, that the six-port junction, designed as a phase discriminator, has the best qualities for demodulating an RF signal.

\section{References}

1. Engen GF. The six-port reflectometer: an alternative network analyser. IEEE Transaction on Microwave Theory and Technique. 1977, 25 (12), pp. 1075-1080.

2. Hoer CA. A network analyser incorporating two six-port reflectometers. IEEE Transaction on Microwave Theory and Techniques. 1977, 25 (12), pp. 1070-1074. 
3. Six-port Measurement Technique: Principles, Impact, Applications. http://www.s-team.sk/download/ SixPortTechnique.pdf. Date accessed: 2008.

4. Marsan E, Schiel JC, Brehm G, Wu K, Bosisio RG. Highspeed carrier recovery circuit suitable for direct digital QPSK transceivers. IEEE Radio and Wireless conference. 2002, pp. 103-106.

5. Gutierrez Miguélez C, Huyart B, Bergeault E, Ja LP. A new automobile radar based on the six-port phase/frequency discriminator. IEEE Transaction on Vehicular Technology. 2000, 49 (4), pp. 1416-1423.

6. Moldovan E, Tatu SO, Gaman T, Wu K, Bosisio RG. A new $94 \mathrm{ghz}$ six port collision avoidance radar sensor. IEEE Transactions on Microwave Theory and Techniques. 2004, 52 (3), pp. 751-759.

7. Li J, Bosisio RG, WU K. Modeling of the six-port discriminator in a microwave direct digital receiver. Canadian Conference on Electrical and Computer Engineering. 1995, 2, pp. 1164-1165.

8. Tatu SO, Denidni T. Analysis of Multipath Fading for Six- port Receiver. Microwave and Optical Technology Letters. 2004, 41 (2), pp. 137-141.
9. Tatu SO, Moldovan E, Wu K, Bosisio RG, Denidni TA. Ka-band analog front end for software defined direct conversion receiver. IEEE Transactions on Microwave Theory and Techniques. 2005, 53 (9), pp. 2768-2776.

10. $\mathrm{Xu} \mathrm{X}$, Bosisio RG, Wu K. Analysis and implementation of six- port software-defined radio receiver platform. IEEE Transactions on Microwave Theory and Techniques. 2006, 54 (7), pp. 2937-2943.

11. Peng H, Yang T, Yang Z. Design and implementation of an ultra wideband six-port network. Progress in Electromagnetics Research. 2012, 131, pp. 293-310.

12. Moscosso-Martir A, Molina-Fernández I. Six-port junction with complete UWB coverage in Multilayer technology. European Microwave Conference. 2011, pp. 655-658.

13. Hussaain R, Sharawi MS. Compact Low Frequency Six-port Design for Wireless Communication Devices. Mediterranean Electrotecnical Conference beirout Melecon. 2014, pp. 29-32.

14. Lakhdhar S, Harabi F, Gharsallah A. A Novel SIW Six-Port Junction. Mediterranean Microwave Symposium (MMS). 2015, pp. 1-5. 